\begin{tabular}{|c|l|}
\hline Title & Does Swall lowing Function Recover in the Long Term in Patients W ith Surgically Treated Tongue Carcinomas? \\
\hline Author(s) & Tei, Kanchu; Sakakibara, Noriyuki; Y amazaki, Y utaka; Ohiro, Y oichi; Ono, Mitsunobu; Totsuka, Y asunori \\
\hline Citation & $\begin{array}{l}\text { Journal of Oral and Maxillofacial Surgery, 70(11), 2680-2686 } \\
\text { https://doi.org/10.1016/.joms.2012.01.021 }\end{array}$ \\
\hline Issue Date & 2012-11 \\
\hline Doc URL & http://hdl.handle.net/2115/50901 \\
\hline Type & article(author version) \\
\hline File Information & JOMS70-11_2680-2686.pdf \\
\hline
\end{tabular}

Instructions for use 


\section{Does The Swallowing Function Recover During The Long-Term In Patients With Surgically Treated Tongue Carcinomas?}

Kanchu Tei, DDS, phD*, Noriyuki Sakakibara, DDS, $\mathrm{phD}^{\dagger}$, Yutaka Yamazaki, DDS, $\mathrm{phD}^{\ddagger}$, Yoichi Ohiro, DDS, $\mathrm{phD}^{\S}$, Mitsunobu Ono, DDS ${ }^{\S}, \mathrm{phD}$ and Yasunori Totsuka, DDS, $\mathrm{phD}^{/}$

Oral and Maxillofacial Surgery, Graduate School of Dental Medicine, Hokkaido University, Sapporo, Japan.

*Associate Professor, Department of Oral and maxillofacial Surgery

$\dagger$ chief, Oral and maxillofacial surgery, Nikko Memorial Hospital

Assistant Professor, Department of Oral Diagnosis and Oral Medicine

$\S$ Assistant Professor, Department of Oral and maxillofacial Surgery

// Professor, Department of Oral and maxillofacial Surgery

KEY WORDS: swallowing function, videofluoroscopic examination, Oropharyngeal Swallow Efficiency (OPSE), long-term follow-up, tongue carcinoma

\section{RUNNING HEAD:}

Post-surgical swallowing function in patients with tongue carcinomas.

\section{CORRESPONDENCE TO:}

Kanchu Tei, Associate Professor

Oral and Maxillofacial Surgery, Graduate School of Dental Medicine,

Hokkaido University

Kita-13, Nishi-7, Kita-ku, Sapporo Japan $\bar{T} 060-8586$

e-mail: teik@den.hokudai.ac.jp

TEL\&Fax 81-11-706-428 


\begin{abstract}
Purpose: The present study aims to measure the post-surgical swallowing function in patients five years after operative treatment of tongue carcinoma.

Patients and Methods: Using a retrospective cohort study design, the investigators enrolled post-surgical patients treated for tongue carcinomas in Hokkaido University Hospital. The primary outcome variable was the Oropharyngeal Swallow Efficiency (OPSE) determined by videoflluoroscopic evaluation and the OPSE at present was compared with that at discharge. Other variables included present nutritional status (Body Mass Index: BMI, Serum Albumin), dietary intake, self-rating of the present swallowing function, and the occurrence of pneumonia. Statistical analysis used the paired t-test and Spearman's rank correlation.

Results: The swallowing function was assessed in 20 patients (11 males and 9 females) subjected to surgical treatment of tongue carcinomas; the median age was 70 years (range 56-90 years) at the present evaluation. The mean values of the liquid OPSE and paste OPSE at present were $26.6 \pm 21.2$ and $21.9 \pm 22.5$ respectively. The mean values of BMI and serum albumin at present were $22.2 \pm 3.4$ and $4.5 \pm 0.3 \mathrm{~g} / \mathrm{dl}$ respectively. All patients had a full oral intake of foods, with a mean self-rated value of $6.4 \pm 2.5$, an acceptable value as evaluated by the patients.

Pneumonia requiring hospitalization had not occurred in these patients.

Conclusions: Long-term follow-up of patients following operative treatment of tongue carcinomas demonstrate acceptable levels of oral function and nutritional status despite objective measures of poor swallowing efficiency assessed using a videofluoroscopy.
\end{abstract}




\section{Introduction}

Evaluation of the post-surgical swallowing function has been performed mainly with videofluoroscopic measurements ${ }^{1-12}$. The post-surgical swallowing function observed with videofluoroscopic measurements ${ }^{1-11}$ has been evaluated with relatively short-term follow-up periods, such as follow-up surveys within one year of the treatment. Because oral cancer patients are a high risk category both for developing local recurrences, regional or distant metastasis, as well as for developing second primary cancers, long-term periodic follow-up is essential for these patients. Only few documents have reported on the post-surgical swallowing function after long-term follow-up ${ }^{12-15}$. Some authors have reported on the post-surgical swallowing function in long-term follow-up surveys using subjective measures such as questionnaires ${ }^{13,14}$ or telephone interviews ${ }^{15}$. Due to limitations as reported by Pauloski ${ }^{5}$, there are few reports of the post-surgical swallowing function in long-term follow-up studies with videofluoroscopic methods. Recently, Nguyen ${ }^{12}$ evaluated 25 head and neck cancer patients with repeated videofluoroscopic measures where the mean follow-up period was 26 months, ranging from 15 to 82 months. Although the swallowing function of these patients was evaluated after the oncologic intervention and using videofluoroscopic measurements for patients with head and neck cancers, there was heterogeneity in the group studied and differences in the treatment modalities for the 25 patients.

The purpose of this study was to establish the post-surgical swallowing function in a long-term follow-up study in patients with tongue carcinomas. The investigators hypothesize that the post-surgical swallowing function evaluated by the videofluoroscopic examination did not recover during the long-term follow-up. The specific aims of the study were to compare the present Oropharyngeal Swallow Efficiency (OPSE) with that at discharge and with that has been 
reported in other follow-up studies ${ }^{7,16,17}$, to compare the present nutritional status (BMI and serum albumin) and food consistency of the current eating to that at discharge, to determine a self-rating of the present swallowing function, and the occurrence of pneumonia. Further, the occurrence of penetration and aspiration evaluated by the videofluoroscopic examination were also determined.

\section{Patients and Methods}

Study design / patients

To address the research purpose, the investigators designed and implemented a retrospective cohort study. The study population was composed of all patients with tongue carcinomas receiving 40Gy of pre-operative radiotherapy and treated with oral and oropharyngeal resection and reconstructed with vascularized free flaps or pedicled flaps between Jan. 1995 and Dec. 2001 at the Department of Oral and Maixillofacial Surgery, Hokkaido University Hospital. To be included in the study sample, patients had to have been followed for more than five years. Patients were excluded as study subjects if they had received post-operative radiotherapy or other surgical interventions due to primary or cervical metastasis after the initial surgery. Patients who had experienced strokes, Parkinson's disease, neuromuscular diseases, or other complaints that could influence the swallowing function after the initial surgery were excluded from the study. Patients were also excluded if they did not agree to enroll in the present study due to difficulty of access, age, or for other reasons. Ethical approval was secured from the Hokkaido University Ethics Committee, and informed consent was obtained in writing from each patient.

Variables 
In the present study, the predictor variable was time, the situation at discharge vs. that at present. The primary outcome variable was the present OPSE determined by videofluoroscopic examination compared with the OPSE those at discharge or with reported OPSE values. Other outcome variables included the present nutritional status (Body Mass Index: BMI, Serum Albumin) and present dietary intake (food consistency of current eating) compared with that at discharge, self-rating of the present swallowing function (using a Visual Analogue Scale: VAS), and a determination of the occurrence of pneumonia. Further, the occurrence of penetration and aspiration evaluated by the videofluoroscopic examination were also determined. The study variables were age, gender, months after initial surgery, clinical stage of tumors at the time of the surgery, tongue resection (partial glossectomy, hemiglossectomy, subtotalglossectomy), neck dissection (unilateral, bilateral), mandibulectomy, the patient using or not using dentures, and participation in post-operative swallowing therapy.

Data collection

The videofluoroscopic examination at the time of the present evaluation was performed between April and June 2007 and the BMI, serum albumin, dietary intake, occurrence of pneumonia, and self-rating of the swallowing function were assessed simultaneously. The BMI, serum albumin and dietary intake at the time of the present evaluation were compared to these parameters at the time of the discharge following the surgery.

In the videofluoroscopic examination, patients were asked to complete two swallows each of 3cc barium liquid and a 1/2 teaspoon of barium paste (total four swallows) in a sitting position. No head stabilizing device was used, and the videofluoroscopic examination was performed during the swallowing in a lateral projection. The results were recorded on videotape and converted to digital data. The oral transit time, pharyngeal transit time, and percent of the 
bolus swallowed were determined with Primere ${ }^{\circledR}$ software (Adobe Systems Incorporated, San Jose, U.S.A.) and the oropharyngeal swallow efficiency (OPSE) was calculated. The OPSE was calculated as percent of the bolus swallowed divided by the time needed to swallow the bolus(Table 1). The method for calculating the oropharyngeal swallow efficiency (OPSE) was according to that reported by Logemann et al. ${ }^{16,17}$. To verify the reliability of the OPSE determined in the present study, ten randomly chosen data sets were reanalyzed by two of the authors. The Pearson correlation coefficients of interobserver reliability were .91 for the liquid and .98 for the paste.

The nutritional status was evaluated using the Body Mass Index (BMI) and serum albumin. The BMI is commonly used to estimate nutritional conditions and calculated as body weight $(\mathrm{kg}) /\{\text { height }(\mathrm{m})\}^{2}$. A score below 18.5 is considered underweight, 18.5-25 as normal weight, 25.0-29.9 as overweight, and 30.0 and above as obese. Values of serum albumin below 4.0 are considered to show malnutrition. Patients were asked to rank the consistency of currently eaten food as regular masticated (no restriction), soft masticated, pastes/ purees, thick liquids and thin liquids. These results were compared to those at the time of discharge. The patients were also asked to rate the present swallowing function compared to that before surgery using Visual Analogue Scale (Rate 0 to 10). If the present swallowing function is equal to that before surgery, the rating is 10 . The occurrence of pneumonia was established by questions to the patients. The penetration and aspiration were determined by the videofluoroscopic examination. Penetration was defined as entry of liquid or paste into the larynx at some level down to but not below the true vocal cords. Aspiration was defined as entry of liquid or paste into the airway below the true vocal cords. The details of the evaluation used in the present study are shown in Table 1. 
Data analysis

The values of the liquid OPSE, Paste OPSE, BMI and serum albumin were analyzed using paired t-tests, and $\mathrm{P}$ values below .05 were considered statistically significant. The correlation between the OPSE and the self-rated swallowing function (VAS) was analyzed using Spearman's rank correlation.

\section{Results}

Between Jan. 1995 and Dec. 2001, 43 patients with tongue carcinomas underwent oro-mandibular reconstruction with vascularized free flap or pedicled flap. Of the 43 patients, 16 patients with primary or cervical lymph node recurrences were excluded from the study here because additional surgery or radiotherapy had been performed after the initial surgery, and seven patients were excluded because they did not agree to be enrolled in the present study due to difficulty of access or age. The study sample then consisted of 20 patients with a median age of 70 years. They were 11 males and 9 females. The stages of the cancers were: 5 of stage II, 11 of stage III, and 4 cases of stage IV. The extent of the tongue surgery, the type of neck dissection performed, and the flap origin are shown in Table 2. All patients had received pull through or commando operations. Segmental resection of the mandible was performed in one patient and marginal resection was performed in two patients. Although the precise volume of the dissected tissue was not estimated in the present study, the approximate volume could be estimated from the type of tongue dissection and the $\mathrm{T}$ stage of each tumor. To increase the motility of the oral structure and to prevent aspiration, all patients had taken part in post-operative swallowing therapy and 4 patients also had received pre-operative swallowing therapy. The swallowing therapy had included tongue range-of-motion exercises and supraglottic swallow maneuvers. Eighteen patients had partial or complete dentures and two patients did not have dentures. Of 
these two patients, one had 28 teeth and the other was edentulous. Six of the 20 patients had also received videofluoroscopic examinations at the time of discharge.

The mean liquid OPSE at present was $26.6 \pm 21.2 \% / \mathrm{sec}$ and 16 patients showed low OPSE. The mean paste OPSE at present was $21.9 \pm 22.5 \% / \mathrm{sec}$ and 16 patients showed low OPSE. Altogether, 13 patients showed low OPSE both in the liquid and paste swallowing, 3 patients showed low OPSE in liquid swallowing and 3 other patients showed low OPSE in paste swallowing. One patient showed good OPSE both in the liquid and paste swallowing. Penetration was established in 8 patients (40\%) with liquid swallowing and in 5 patients $(25 \%)$ with paste swallowing. Aspiration was established in three patients (15\%) with liquid swallowing and one patient (5\%) in paste swallowing. One patient showed aspiration both in liquid and paste swallowing. The extent of the tongue resections for these three patients with aspiration were partial glossectomy, hemiglossectomy, and subtotalglossectomy, respectively. The 6 patients who underwent videofluoroscopic examinations at hospital discharge after the surgery, had a mean liquid OPSE of $15.9 \pm 18.7 \% / \mathrm{sec}$ and the mean for the paste OPSE was 8.8 $\pm 7.4 \% / \mathrm{sec}$. The mean liquid OPSE at present were not statistically significantly different from that at discharge $(\mathrm{p}=0.4)$. However, the mean paste OPSE at present was higher than that discharge $(\mathrm{p}=0.046)$.

The mean BMI of all the participating patients at the hospital discharge after the surgery was $21.0 \pm 2.9$. Thirteen patients were classified as having normal weight, five patients were underweight, and 2 patients were overweight. The mean BMI at the time of the present evaluation was $22.2 \pm 3.4$. At the present survey, 13 patients were classified as having normal weight, three patients as underweight, and 4 patients as overweight. In five patients who were classified as underweight at the hospital discharge, two patients now showed normal weight and 
three patients remained underweight. There was no statistically significant difference between the BMI at discharge and the BMI at present $(\mathrm{p}=0.1142)$. The mean serum albumin at the post-surgical hospital discharge and at the present evaluation were $4.4 \pm 0.4 \mathrm{~g} / \mathrm{dl}$ and $4.5 \pm 0.3$ $\mathrm{g} / \mathrm{dl}$, respectively. There was no statistically significant difference between the serum albumin at the discharge and the serum albumin at present $(\mathrm{p}=0.6864)$. Three patients with values of serum albumin below 4.0 at the hospital discharge registered above 4.0 at the present evaluation. The three patients with BMI showing them to be underweight at the present evaluation, all had serum albumin values above $4.0 \mathrm{~g} / \mathrm{dl}$.

All patients ingested food without tube feeding at the time of the present evaluation. The food consistency ingested by the patients at the post-surgery hospital discharge was: 1 patient with regular masticated (no restriction), 8 patients with soft masticated, 3 patients reported ingesting paste / purees, 8 patients reported ingesting thick liquids. The food consistency ingested at the time of the present evaluation was: 12 patients with regular masticated, 6 with soft masticated foods, and 2 with pastes / purees. In total, 16 patients showed improvements, three had remained unchanged, and one patient showed a deterioration in the consistency of the ingested food.

The mean value of the self-rating of the present swallowing function was $6.4 \pm 2.5$, 7.4 \pm 3.4 for the partial glossectomy subgroup $(n=7), 5.8 \pm 2.1$ for the hemiglossectomy $(n=9)$, and $6.0 \pm 1.2$ for the subtotal glossectomy $(\mathrm{n}=4)$ subgroup. There was no statistically significant correlation between the OPSE at present (liquid, paste) and VAS ( $r=0.108, r=0.373)$. Pneumonia which had lead to hospitalization had not occurred among these twenty patients during the period between the discharge after the surgery and the present investigation.

\section{Discussion}


The purpose of this study is to establish the post-surgical swallowing function in a long-term follow-up study in patients with tongue carcinomas conducted more than five years after surgery. The investigators hypothesize that the post-surgical swallowing function evaluated by videofluoroscopic examination had not recovered at the long-term follow-up. The specific aims of the study were to compare the present OPSE with the OPSE at discharge and with reported OPSE values, to compare the present nutritional status (BMI and serum albumin) and the consistency of currently ingested food to that at discharge, to determine the self-rating of the present swallowing function, and the occurrence of pneumonia post-surgically. Further, the occurrence of penetration and aspiration evaluated by videofluoroscopic examination were also determined.

The post-surgical swallowing function measured with OPSE did not recover fully in patients with tongue carcinomas at the long-term follow-up investigation. Although the mean value of paste OPSE was higher than at discharge, this value is still lower than that before surgery or the level in healthy humans. Also the number of patients who underwent videofluoroscopic examinations at discharge was small compared to the total numbers of patients in the present study. The OPSE is a convenient measure to compare clinical populations that may have different impairments in oropharyngeal swallowing ${ }^{17}$, and it has been employed in the evaluation of post-surgical swallowing dysfunction of patients with head and neck cancers $^{1-5,7,8}$. In the rather short follow-up period reports ${ }^{1-5,7,8}$, it has been concluded that OPSE does not recover to the level before the surgery, after up to one-year. The average value of OPSE in healthy humans has been reported as $40-50 \%^{16,17}$. Therefore OPSE values below $40 \%$ were considered low OPSE in the present study. Further, the average post-operative OPSE values in patients with oral cancer in a 1-year follow-up study have been reported to be around 
$20 \%{ }^{7}$ which is close to the values established in the present study. Therefore, the results of the present study indicate that the Oropharyngeal Swallow Efficiency (OPSE) in patients with tongue carcinomas had not recovered fully at the median time of 104 months following treatment (range 63-147 months).

Long-term follow-up is crucial for patients with head and neck cancers ${ }^{6}$, however, there are only few reports concerned with this issue ${ }^{12-15}$. Speksnijder ${ }^{15}$ assessed the self-perceived oral function in 69 patients with oral cancers 5 years post-operatively using telephone interviews, and concluded that patients with tongue carcinomas experienced deterioration in the dental status, chewing ability, and xerostomia, when compared with the level before the oncologic intervention. Freidlander ${ }^{13}$ evaluated 26 patients with tongue base resections after a mean follow-up duration of 8.6 years using questionnaires including the Performance Status Scale for head and Neck Cancer (PSS) and the Karnofsky Performance Status Scale (KPS). They concluded that the long-term functional status of these patients who had undergone resection of a significant portion of the base of the tongue was good. Yun ${ }^{14}$ assessed post-surgical swallowing function of 14 patients who had undergone total glossectomy 1-7 years previously using a 7-point scale and concluded that all the patients ingested soft food at the follow-up survey. Due to restrictions like monetary or patient access that are mentioned by Pauloski ${ }^{5}$, outcomes of the videofluoroscopic evaluations of post-surgical swallowing function more than a few years after surgery has rarely been studied. Recently, Nguyen et al ${ }^{12}$ evaluated 25 patients with head and neck cancers with repeated videofluoroscopic examinations, here the mean period from the surgery was 26 months, ranging from 15 to 82 months. It was concluded that the swallowing function had not recovered in $68 \%$ of the patients. In the Nguyen et al report, the swallowing function was evaluated after oncologic intervention using 
videofluoroscopic examinations for patients with head and neck cancers, there was heterogeneity in the group studied and in the treatment modalities of these 25 patients, making the present study more focused and applicable to an evaluation of post-surgical swallowing functioning in patients with tongue carcinomas.

In the long term follow-up study reported here, the nutritional status of the patients was adequate and all patients had full oral intakes at the time of the present evaluation. The food consistency of currently eaten was markedly improved compared to that at the time of discharge. The BMI and serum albumin were used to evaluate the nutritional status because these indexes are simply established and are in common use worldwide. Except for 3 patients with low BMI at the time of discharge, 17 patients maintained good nutritional status as evaluated by the BMI. The level of serum albumin was above $4.0 \mathrm{~g} / \mathrm{dl}$ in all the patients. These results indicate that the patients in the present study have maintained good nutritional status for more than five years post-surgically. For the oral food intake, the food consistency or normalcy of diet within 1 year, post-surgery for patients with head and neck cancers, $22-86 \%$ have been reported to eat normal diets and to ingest regular masticated or soft foods $s^{6,7,913,18}$. The long-term status, 8.6 years (range 2.5-17.5years) post-surgically, was reported by Friedlander ${ }^{13}$, who evaluated 26 patients who had undergone tongue base resection by the Karnofsky Performance Scale. The mean score of the normalcy of diet was 79.8 showing it to be all meat. In the present study, $90 \%$ of the patients ingested regular masticated or soft chewable foods at the time of the present evaluation and the food consistency was markedly improved compared with that at the time of discharge.

Twelve patients rated their swallowing function as generally acceptable (VAS 7.0 and over). The results of the present study shows that the OPSE is inconsistent with the dietary level and self-rating of the present swallowing function reported by the participants in the study here. 
Reasons for the disagreement may be that the videofluoroscopic examination has limitations such as in the amount, texture, temperature, and taste of test foods. Further, patient posture or surroundings when eating are different from that at the everyday settings the patients are accustomed to, and at the videofluoroscopic examination, the patients are asked to swallow at the command of the researcher. These patients may be considered to be able to ingest orally, perhaps by diet modifications for safer swallowing or by adjusting the swallowing posture, adjusting the amount of a mouthful of food and liquid or by finding a more appropriate place to put the food in the mouth where patients can display optimum swallowing performance to overcome the disadvantages shown by the videofluoroscopic evaluation. Additionally, the effort made by the post-surgical oral cancer patients may also be related to discrepancies between the perceptions of the swallowing and the swallowing function for the group ${ }^{19}$. In conclusion, the apparent disagreement in the results of the videofluoroscopicaly-confirmed swallowing function and the results of the self-rating of the present swallowing function, makes it possible to postulate that the relative high self-rating values recorded here reflects real improvements in the dietary level or food consistency of the eating of these patients.

Aspiration has been reported in up to $44 \%$ of patients with surgically treated oral and oropharyngeal cancers with free-flap reconstruction, in follow-up studies within one year of the treatment ${ }^{6}$, and the incidence of aspiration in patients treated with chemoradiation was reported as somewhat higher ${ }^{9}$. Previously, we have reported the incidence of aspiration as $12 \%$ for liquid and $4 \%$ for paste swallowing for patients with oral carcinomas treated with preoperative radiation and surgery, at a 1 year post-intervention survey ${ }^{7}$. In the present study, the incidence of aspiration was $15 \%$ for liquid and $5 \%$ for paste swallowing. These results suggest that the incidence of aspiration does not increase long-term in these patients, where no other 
intervention has taken place. None of the patients had experienced pneumonia after discharge regardless of poor swallowing function confirmed by the videofluoroscopic examination. The reports of pneumonia were obtained from patient reflections, but it appears highly likely that the patients had suffered no pneumonia serious enough to require hospitalization. Since swallowing dysfunction causes aspiration, a relationship between swallowing dysfunction and aspiration pneumonia would appear highly likely, but there is no consistent evidence of this ${ }^{20-22}$. Further, in clinical settings we have found that some patients who aspirate do not develop pneumonia, and that there are other patients with similar conditions who do develop aspiration pneumonia. The patients in the present study showed good nutritional states and medical status maintaining the immune system well and this may have prevented the occurrence of pneumonia induced by aspiration problems.

The present study evaluated the post-operative swallowing function with a videofluoroscopic examination in a uniform set of 20 patients with tongue carcinomas. The treatment modalities of the patients were similar and the swallowing function measured with OPSE was evaluated more than five years after the surgical procedure as a long-term follow-up evaluation. The results indicate that post-surgical swallowing function measured with OPSE did not recover during the period until the follow up reported here. However, the results of the present study were determined by the retrospective study design, therefore to assess the post-surgical swallowing function after a further five years with the sample of the present study would be relevant to support the results of the present study. Besides, the number of the patients enrolled in the present study was small, therefore differences in the post-surgical swallowing function in patients with tongue carcinomas was not assessed based on site and size of resection. Further prospective cohort study is needed to assess differences in swallowing function in 
long-term follow-up studies based on site and size of resection in patients with tongue carcinomas.

The results of the present study showed that patients with tongue carcinomas more than 5 years post-surgically displayed good nutritional and medical status and had an acceptably norrmal oral intake regardless of poor swallowing function established by videofluoroscopic evaluation. Because the post-surgical swallowing function does not recover to the level before the surgery, the goals of the functional recovery should be set to maintain an acceptable medical status and to avoid aspiration pneumonia ${ }^{7}$.

\section{References}

1. Pauloski BR, Logemann JA, Rademaker AW: Speech and swallowing function after anterior tongue and floor of mouth with distal flap reconstruction. J Speech Hear Res $36: 267-276,1993$

2. Logemann JA, Pauloski BR, Rademaker AW: Speech and swallow function after tonsil / base of tongue resection with primary closure. J Speech Hear Res 36 : 918-926, 1993

3. Pauloski BR, Logemann JA, Rademaker AW: Speech and swallowing function after oral and oropharyngeal resections : one year follow up. Head Neck 16 : 313-322, 1994

4. McConnel FMS, Pauloski BR, Logemann JA: Functional results of primary closure vs flaps in oropharyngeal reconstruction. Arch Otolaryngol Head Neck Surg 124 : 625-630, 1998

5. Pauloski BR, Rademaker AW, Logemann JA: Surgical variables affecting swallowing in patients treated for oral / oropharyngeal cancer. Head neck 26: 625-636, 2004

6. Markkanen-Leppänen M, Isotaro E, Mäkitie AA: Swallowing after free-flap reconstruction 
in patients with oral and pharyngeal cancer. Oral Oncology 42: 501-509, 2006

7. Tei K, Maekawa K, Kitada H: Recovery of post-surgical swallowing function in patients with oral cancer. J Oral maxillofac Surg 65:1077-1083, 2007

8. Borggreven PA, Leeuw IV, Rinkel RN: Swallowing after major surgery of the oral cavity or oropharynx: A prospective and longitudinal assessment of patients treated by microvascular soft tissue reconstruction. Head Neck 29: 638-647, 2007

9. Rieger JM, Zalmanowitz JG., Li SYY: Functional outcomes after surgical reconstruction of the base of the tongue using the radial forearm free flap in patients with oropharyngeal carcinoma. Head Neck 29: 1024-1032, 2007

10. Brown L, Rieger J M, Harris J: A Longitudinal Study of Functional Outcomes After Surgical Resection and Microvascular Reconstruction for Oral Cancer: Tongue Mobility and Swallowing Function. J Oral Maxillofac Surg 68: 2690-2700, 2010

11. Archontaki M, Athanasiou A, Starvrianos SD, et al.:Functional results of speech and swallowing after free flap reconstruction, Eur Arch Otorhinolaryngol 267:1771-1777,2010

12. Nguyen NP, Moltz CC, Frank C: Evolution of chronic dysphagia following treatment for head and neck cancer. Oral Oncology 42: 374-380, 2006

13. Friedlander P, Caruana S, Singh B: Functional status after primary surgical therapy for squamous cell carcinoma of the base of the tongue. Head Neck 24: 111- 114, 2002

14. Yun IS, Lee DW, Lee WJ, et al: Correlation of neotongue volume changes with functional outcomes after long-term follow-up of total glossectomy, J Craniofac Surg 21: 111-16, 2010

15. Speksnijder CM, Glas HW, Bilt Andries: Oral function after oncological intervention in the oral cavity: A retrospective study. J Oral Maxillofac Surg 68:1231-1237, 2010

16. Logemann JA, Kahrilas PJ: Relearning to swallow after stroke- application of maneuvers 
and indirect biofeedback: A case study. Neurology 40: 1136-1138, 1990

17. Rademaker AW, Pauloski BR, Logemann JA: Oropharyngeal swallow efficiency as a representative measure of swallowing function. J. Speech Hearing Res. 37: 314-325, 1994

18. Schache AG., Lieger O, Rogers P: Predictors of swallowing outcome in patients treated with surgery and radiotherapy for advanced oral and oropharyngeal cancer. Oral oncology 45: 803-808, 2009

19. Pauloski BR, Rademaker AW, Logemann JA: Swallow function and perception of dysphagia in patients with haed and neck cancer. Head Neck 25: 555-565, 2002

20. Langmore SE, Terpenning MS, Schork A et al: Predictors of aspiration pneumonia: how important is dysphagia? Dysphagia 13: 69, 1998.

21. Feinberg MJ, Knebl J, Tully J: Prandial aspiration and aspiration pneumonia in an elderly population followed over 3years. Dysphagia 11: 104-109, 1996

22. Langmore SE, Skarupski KA, Park PS et al: Predictors of aspiration pneumonia in nursing home residents, Dysphagia 17: 298, 2002.

\section{Tables: No legend}

\section{Table 1. Details of evaluation}

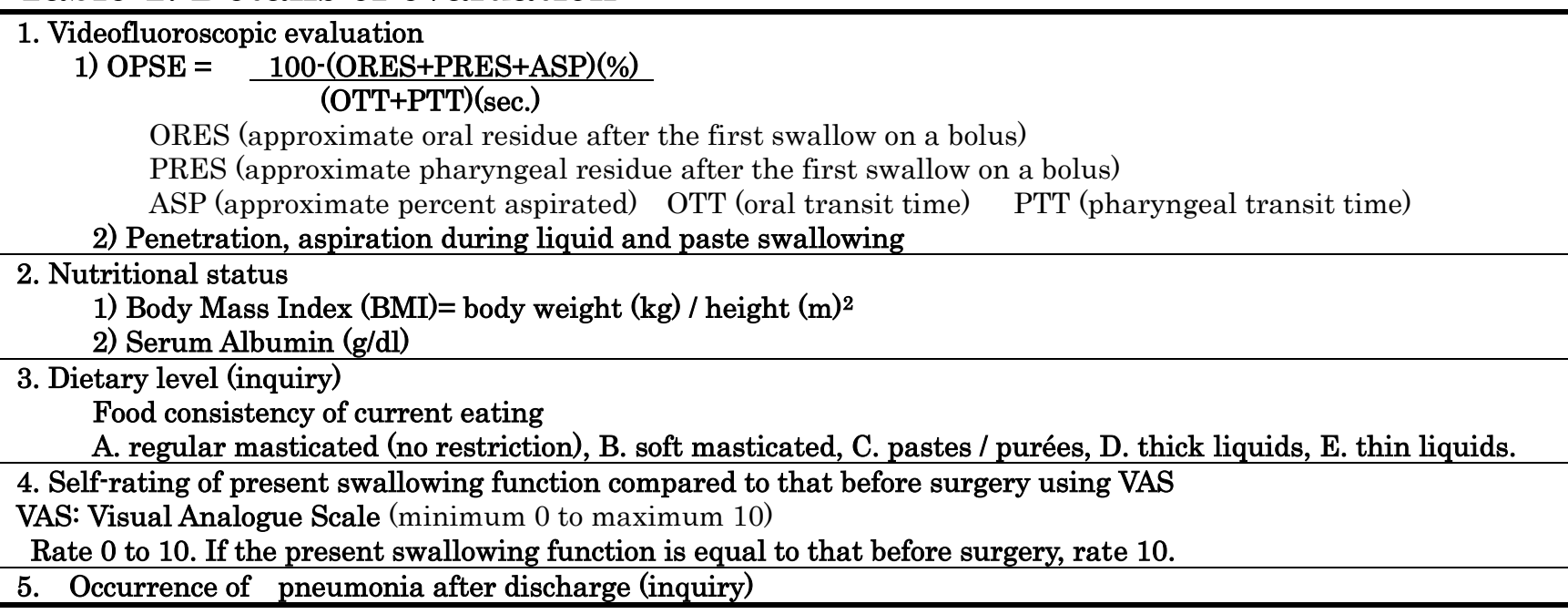




\section{Table2. Particulars of patients}

\begin{tabular}{ll}
\hline Age & $56-90$ (median : 70) \\
Male : Female & $11: 9$ \\
Months after initial surgery & $63-147$ months (median: 104 months ) \\
Clinical stage of tumors at & T2: 10, T3: 6, T4: 4 \\
surgery & Stage II: 5, stage III: 11, stage IV: 4 \\
Preoperative treatment & 40 Gy of preoperative radiotherapy \\
Tongue resection & subtotalglossectomy: 4 hemiglossectomy:9 partial glossectomy: 7 \\
Neck dissection & Unilateral:16, bilateral:4 \\
Used flaps & Vascularized free flap 11 (RA: 3, FA: 8) \\
& Pedicled Flap 9 (DP: 4, PMMC: 3,cervical: 2) \\
\hline
\end{tabular}

RA:rectus abdominis flap FA: forearm flap DP: deltopectoral flap

PMMC: pectoralis major myocutaneous flap

\section{Table 3 The values of the liquid OPSE, Paste} OPSE, BMI, Serum Albumin and VAS

\begin{tabular}{lccr}
\hline Measurements & at discharge & at present $(\mathrm{n}=20)$ & p value \\
\hline liquid OPSE (\%/sec.) & $15.9 \pm 18.7(\mathrm{n}=6)$ & $26.6 \pm 21.2$ & .400 \\
paste OPSE (\%/sec.) & $8.8 \pm 7.4(\mathrm{n}=6)$ & $21.9 \pm 22.5$ & .046 \\
BMI & $21 \pm 2.9(\mathrm{n}=20)$ & $22.2 \pm 3.4$ & .114 \\
Serum Albumin (g/dl) & $4.4 \pm 0.4(\mathrm{n}=20)$ & $4.5 \pm 0.3$ & .686 \\
VAS & $\mathrm{NA}$ & $6.4 \pm 2.5$ & $\mathrm{NA}$ \\
\hline
\end{tabular}

NA: Not Available

values shown are in mean $\pm \mathrm{SD}$. 